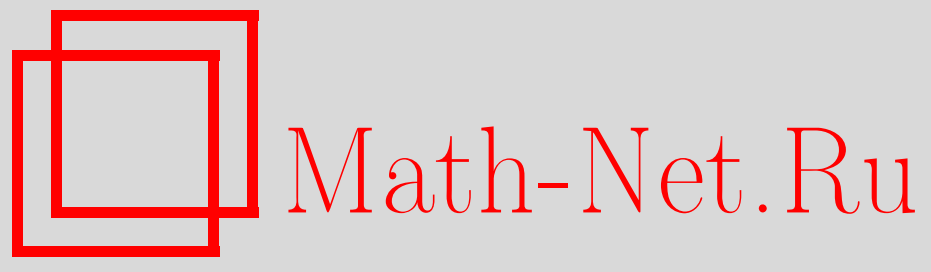

Е. М. Вечтомов, О. В. Старостина, Структура абелеворегулярных положительных полуколец, УМH, 2007, том 62, выпуск 1, 199-200

DOI: https://doi.org/10.4213/rm5584

Использование Общероссийского математического портала Math-Net.Ru подразумевает, что вы прочитали и согласны с пользовательским соглашением http://www . mathnet.ru/rus/agreement

Параметры загрузки:

IP : 54.198 .187 .58

26 апреля 2023 г., 13:24:56

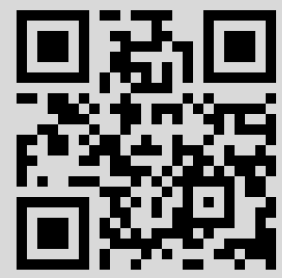




\section{Структура абелево-регулярных положительных полуколец}

\section{Е. М. Вечтомов, О. В. Старостина}

1. Полукольцо $S$ называется абелево-регулярным положительным или arp-nолукольцом, если $S$ - положительное (т.е. элемент $a+1$ обратим в $S$ для любого $a \in S$ ) и абелево-регулярное полукольцо (т.е. для каждого $a \in S$ уравнение $a x a=a$ разрешимо в $S$ и любой его идемпотент $е$ перестановочен со всеми элементами из $S$ ) [1]. Основные понятия и структурная теория класса arp-полуколец развиты в работе [1].

Пусть $L(S)$ - множество всех идемпотентов $\operatorname{arp-полукольца~} S$, а $U(S)$ - множество всех обратимых элементов в $S$. Изучение аrр-полуколец сводится к изучению троек $\left\langle L(S), U(S), \varphi_{S}\right\rangle$.

$\langle U(S),+, \cdot\rangle$ - полутело без нуля относительно операций сложения и умножения в $S .\langle L(S), \vee, \cdot\rangle$ - дистрибутивная решетка с 0 и 1 относительно операции умножения · полукольца $S$ и сложения $\vee$. При этом операция сложения $\vee$ определяется следующим образом: так как каждый элемент $a$ arp-полукольца $S$ представляется в виде произведения $a=e_{a} \cdot u$ однозначно определенного идемпотента $e_{a} \in L(S)$ и обратимого элемента $u \in U(S)$, то полагаем $f \vee g=e_{f+g}$. Пусть для $u, v \in U(S)$ $u \varphi(e) v \Leftrightarrow e u=e v$. Отображение $\varphi=\varphi_{S}: e \mapsto \varphi(e)$ решетки $L(S)$ в решетку всех конгруэнций $\operatorname{Con} U(S)$ полутела $U(S)$ является решеточным антигомоморфизмом.

Вводится также категория абстрактных троек $\langle L, U, \varphi\rangle$, состоящих из ограниченной дистрибутивной решетки $L$, полутела без нуля $U$ и решеточного антигомоморфизма $\varphi: L \rightarrow \operatorname{Con} U$, переводящего 0 в 1 и 1 в 0 . Абстрактная тройка, соответствующая некоторому аrр-полукольцу $S$, называется индуцированной.

В теории агр-полуколец возникают следующие естественные вопросы [1]:

1) всякая ли тройка $\langle L, U, \varphi\rangle$ индуцируется некоторым arp-полукольцом?

2) следует ли из изоморфизма индуцированных троек изоморфизм соответствующих агр-полуколец?

В [1] был приведен пример неиндуцированной тройки (пример 3.2) и было установлено, что для индуцированности тройки $\langle L, U, \varphi\rangle$ достаточно, чтобы $\operatorname{Im} \varphi$ содержался в некоторой булевой подрешетке решетки $\operatorname{Con} U$ (теорема 3.3). Построение arp-полукольца по такой тройке $\langle L, U, \varphi\rangle$ осуществляется следующим образом. Пусть

$$
S=\bigcup_{e \in L}^{\bullet} U / \varphi(e)
$$

есть дизъюнктное объединение факторполутел $U / \varphi(e)=\left\{[u]_{\varphi(e)} \mid u \in U\right\}$. Для любых $e \in L$ и $u \in U$ можно отождествить $[1]_{\varphi(e)} \equiv e,[u]_{\varphi(1)} \equiv u$, тогда $[u]_{\varphi(e)}=e u$. Операции умножения и сложения определяются следующим образом: $e u \cdot f v=e f \cdot u v$, $e u+f v=(e \vee f) w$, где $e, f \in L, u, v, w \in U$ и обратимый элемент $w$ удовлетворяет соотношениям $w \varphi(e f)(u+v), w(\varphi(e) \circ \psi(f)) u, w(\varphi(f) \circ \psi(e)) v$.

Второй вопрос был положительно решен для предбулевых arp-полуколец, т.е. для полуколец, у которых $\operatorname{Im} \varphi_{S}$ является булевой подрешеткой в решетке $\operatorname{Con} U$, и для идемпотентных аrр-полуколец [1; теоремы 4.1 и 5.6].

Основная лемма. Для любого агр-полукольца $S$ образ $\operatorname{Im} \varphi_{S}$ вкладывается в булеву подрешетку $\operatorname{Con} U(S)$.

ДоказАтельство. Для любого $e \in L(S)$ дополнением $\varphi(e)$ в решетке $\operatorname{Con} U(S)$ является конгруэнция $\psi(e): u \psi(e) v \Leftrightarrow u+e x=v+e y$ для некоторых $x, y \in U(S)$. Действительно, для любых элементов $u, v \in U(S)$ имеем $u \psi(e)(u+e v) \varphi(e)(u+v)$ и $v \psi(e)(v+e u) \varphi(e)(u+v)$. Откуда $u(\psi(e) \circ \varphi(e)) v$. Если $u, v-$ произвольные элементы полутела $U(S)$ такие, что $u(\psi(e) \cap \varphi(e)) v$, то $e u=e v$ и $u+e x=v+e y$ для некоторых $x, y \in U(S)$. Складывая почленно последние два равенства, получаем $u+e(u+x)=$ $v+e(v+y)$, или $u\left(1+e u^{-1}(u+x)\right)=v\left(1+e v^{-1}(v+y)\right)$. Поскольку $e(u+x)=e(u+e x)=$ 
$e(v+e y)=e(v+y)$ и $e u^{-1}=e v^{-1}$, то $u=v$. Нетрудно убедиться в том, что $\operatorname{Im} \varphi$ и $\operatorname{Im} \psi$ порождают булеву подрешетку в решетке $\operatorname{Con} U(S)$.

Отображение $\psi_{S}: L(S) \rightarrow \operatorname{Con} U(S), e \mapsto \psi(e)$ есть решеточный гомоморфизм.

Учитывая теорему 3.3 из [1], получаем следующие результаты, отвечающие на вопросы 1) и 2).

Теорема 1. Абстрактная тройка $\langle L, U, \varphi\rangle$ является индуцированной тогда и только тогда, когда все элементы $\operatorname{Im} \varphi_{S}$ дополняемы в $\operatorname{Con} U$.

Изоморфизмом индуцированных троек $\left\langle L_{1}, U_{1}, \varphi_{1}\right\rangle$ и $\left\langle L_{2}, U_{2}, \varphi_{2}\right\rangle$ называется паpa $(\beta, \gamma)$ таких изоморфизмов $\beta: L_{1} \rightarrow L_{2}, \gamma: U_{1} \rightarrow U_{2}$, что $u \varphi_{1}(e) v$ равносильно $\gamma(u) \varphi_{2}(\beta(e)) \gamma(v)$

Теорема 2. Два произволъных агр-полуколъца изоморфны тогда и толъко тогда, когда их индуцированные тройки изоморфны.

2. В $[1 ; \S 4]$ было изучено строение конгруэнций на предбулевых полукольцах и доказано, что существует биекция между множеством всех конгруэнций $\rho$ на предбулевом полукольце $S$ и множеством всех согласованных пар $(\sigma, \tau)$ конгруэнций на $L(S)$ и $U(S)$. Этот результат можно уточнить и распространить на произвольные arp-полукольца.

Пусть $\sigma$ - конгруэнция на дистрибутивной решетке $L(S)$ и $\tau$ - конгруэнция на полутеле $U(S)$. Пара конгруэнций $(\sigma, \tau)$ называется согласованной, если она удовлетворяет условию $е \sigma f \Rightarrow \tau \circ \varphi(e)=\tau \circ \varphi(f)$ для любых $e, f \in L(S)$.

ПредлОЖЕНИЕ 1. Бинарное отношение $\rho$ на атр-полукольце $S$ является конгруэниией на $S$ тогда и толъко тогда, когда $\sigma=\left.\rho\right|_{L(S)} u \tau=\left.\rho\right|_{U(S)}-$ конгруэниии на $L(S)$ и $U(S)$ соответственно, образующие согласованную пару $(\sigma, \tau), u($ еи $\rho(f v) \Leftrightarrow$ e $\sigma f u u(\tau \circ \varphi(e)) v$.

Теорема 3. Отображение $\alpha: \rho \mapsto\left(\left.\rho\right|_{L(S)},\left.\rho\right|_{U(S)}\right)$ является изоморбизмом решетки всех конгруэниий Con $S$ агр-полукольца $S$ на подрешетку всех согласованных пар конгруэниий на $L(S)$ и $U(S)$ решетки $\operatorname{Con} L(S) \times \operatorname{Con} U(S)$, более того, Con $S$ является подпрямым произведением решеток $\operatorname{Con} L(S)$ u $\operatorname{Con} U(S)$ u $\alpha(0)=(0,0)$, $\alpha(1)=(1,1)$.

СледСтвиЕ. Решетка конгруэнций Con $S$ произвольного агр-полуколъца $S$ модулярна. Решетка конгруэниий $\mathrm{Con} S$ агр-полукольиа $S$ дистрибутивна тогда и только тогда, когда решетка конгруэнций $\operatorname{Con} U(S)$ полутела $U(S)$ дистрибутивна.

Предполагается, что гомоморфизмы полуколец сохраняют 0 и 1.

Теорема 4. Отображение $\alpha: S \rightarrow T$ является гомоморбизмом агр-полукольца $S$ в агр-полукольио $T$ тогда и только тогда, когда $\beta=\left.\alpha\right|_{L(S)}-$ решеточный гомоморфизм $L(S)$ в $L(T)$, сохраняющий $0 u 1, \gamma=\left.\alpha\right|_{U(S)}$ - гомоморфизм полутела $U(S)$ в полутело $U(T)$ и для любых $е \in L(S), u, v \in U(S)$

$$
u \varphi_{S}(e) v \Rightarrow \gamma(u) \varphi_{T}(\beta(e)) \gamma(v) \quad u \quad u \psi_{S}(e) v \Rightarrow \gamma(u) \psi_{T}(\beta(e)) \gamma(v),
$$

причем $\alpha($ eu $)=\beta(e) \gamma(u)$.

\section{Список литературы}

[1] Е. М. Вечтомов, А. В. Михалев, В. В. Чермных, Труды семинара им. И. Г. Петровского, 20 (1997), 282-309.

\section{Е. М. Вечтомов (E. M. Vechtomov)}

Вятский государственный гуманитарный университет, г. Киров

E-mail: vecht@mail.ru

\section{O. В. Старостина (O. V. Starostina)}

Вятский государственный гуманитарный университет, г. Киров
Представлено А. В. Михалёвым Принято редколлегией 27.10.2006 